Edición Extraordinaria. p.p.1057-1066

Memorias del VIII Encuentro Nacional de Experiencias en Enseñanza de la Biología y la Educación Ambiental. III Congreso Nacional de Investigación en Enseñanza de la Biología.

\title{
"POSIBILIDADES DE RE-PENSAR LA ENSEÑANZA DE LO VIVO Y LA VIDA EN LA ESCUELA: UNA LECTURA EN CLAVE DE LA ESTÉTICA Y LA FORMACIÓN DE MAESTROS DE BIOLOGÍA"
}

\section{"POSSIBILITIES OF RE-THINK TEACHING OF ALIVE AND LIFE AT SCHOOL: A READING IN KEY ESTHETIC AND TEACHER'S TRAINING OF BIOLOGY”'}

\author{
Por: Claudia Jaramillo Sánchez ${ }^{1}$ \\ Deysi Serrato Rodríguez ${ }^{2}$
}

\section{RESUMEN}

El presente escrito da cuenta de algunos hallazgos que conciernen a dos investigaciones ${ }^{3}$ cuyo punto de cruce singular se teje en la problematización de la enseñanza de lo vivo y la vida en la escuela, en clave de lectura de las relaciones entre la estética y la formación de maestros de biología, como una de las condiciones que posibilita preguntarse por las relaciones que el sujeto establece frente al mundo desde matices de sensibilidad, belleza, armonía y contemplación, lo cual no es reductible a lo emocional $y / 0$ afectivo. Estas investigaciones se fundamentan a la luz de la mirada arqueológica-genealógica como posibilidad de visibilizar y poner en cuestión los hallazgos alusivos a ciertas prácticas en relación con lo vivo, la vida y su enseñanza en dos momentos históricos distintos que refieren a los años 1930-1940 y 2000-2012.

PALABRAS CLAVES: Vivo, vida, enseñanza, formación de maestros, estética.

\section{ABSTRACT}

This paper shows some discoveries about two researches whose point of singular intersection is weaved in the problematization about alive and life teaching at school, in key of reading of relationship between esthetic and teacher's training of biology like one of the possible conditions asks for relationships that individual establishes facing towards world from shades of sensitivity, beauty, harmony and contemplation, which is not shrinkable to emotional or affective aspects. These researches are based on light of archaeological-genealogical overview, like possibility of making visible and call into

Licenciada en Biología de la Universidad Pedagógica Nacional. Candidata a Magíster en Educación de la UPN

${ }^{2}$ Licenciada en Biología. Especialista en Pedagogía. Candidata Magíster en Educación. UPN.

3. Las dos investigaciones se constituyen en trabajos de grado desarrollados en el marco de la Licenciatura en Biología y la

Especialización en Pedagogía de la Universidad Pedagógica Nacional. La primera lleva como título "FORMAS Y FUERZAS DE LA

EXPERIENCIA ESTÉTICA: PINCELADAS, MATICES Y POSIBILIDADES EN LA FORMACIÓN DE MAESTROS DE BIOLOGÍA DE

LA UNIVERSIDAD PEDAGÓGICA NACIONAL." y la segunda se denomina "PRÁCTICAS DISCURSIVAS A PROPÓSITO DE LA

BOTÁNICA COMO SABER ESCOLAR ENTRE 1930 Y 1940" 


\section{Edición Extraordinaria. p.p.1057-1066}

Memorias del VIII Encuentro Nacional de Experiencias en Enseñanza de la Biología y la Educación Ambiental. III Congreso Nacional de Investigación en Enseñanza de la Biología.

question discoveries that alluding to some practices in relation with alive and life and its teaching in two different historic moments that refers to the years 1930-1940 y 20002012.

KEY WORDS: Alive, life, teaching, teacher's training, esthetic.

\section{INTRODUCCIÓN: UNA APUESTA DE PENSAMIENTO POR LA ENSEÑANZA DE LO VIVO Y LA VIDA DESDE LA PEDAGOGÍA}

Configurar apuestas de pensamiento deviene en confrontación con nosotros mismos desde las certezas que nos acompañan, deviene según Foucault (1999) en tomar distancia, separarse y desatarse de ello, para constituirlo en problema. Una de las condiciones que incita al pensamiento es la investigación como posibilidad de configuración del sujeto, desde el despliegue de relaciones que dan lugar a la pregunta por la singularidad del mismo, en tanto escenario que sitúa la configuración de diferentes modos de vida. Un devenir que se encuentra cruzado por el cuestionamiento de ser maestro de biología, por las fuerzas que atraviesan su constitución y por el posicionamiento de la enseñanza de lo vivo y sus relaciones con la vida desde diversas ópticas de creación. De ahí que, la inquietud a propósito del lugar de la estética tanto en la formación de maestros de biología como en las opciones que recrean éstos en la escuela, tenga como lugar de emergencia la formación en la Licenciatura en Biología de la UPN, pues la formación se teje entre los intersticios del gobierno de los otros o el deber ser y el gobierno de sí, o aquellas posibilidades que evocan la pregunta por sí mismo, la deconstrucción y creación de otros modos de existencia, la pasión por la lectura, la escritura y el arte como formas de movilización.

Desde esta perspectiva, se asume la enseñanza como un acontecimiento que atraviesa y desborda la relación pedagógica que se teje entre maestro y estudiante, pues de acuerdo con Martínez (2003), ésta tiene que ver con un sujeto que da algo a otro sin un fin determinado diferente a la incitación del pensamiento como ejercicio artístico, estético y ético. Precisamente esta incitación, nos permite asumir la biología no desde su pretensión científica, sino en tanto saber que permite el despliegue y encuentro con otros saberes que potencian su enseñanza, en ese sentido el posicionarse desde el saber, implica abrir la mirada hacia formas de ver y ser en el mundo atravesadas por las relaciones entre lo vivo y la vida. En este mismo entramado, se despliega la pedagogía, distanciándonos de su idea de representación, es decir, aquella que se refiere a un enunciado superfluo y limitado a mostrarle al maestro qué y cómo hacer para constituirse en sujeto modelo diluyendo así la posibilidad de creación en su práctica (Peñuela, 2010), nos interesa más bien, crear hilos de despliegue hacia la pedagogía que posibilita trascender muros y fronteras, desmontando su origen o finalidad, una pedagogía por re- 


\section{Edición Extraordinaria. p.p.1057-1066}

Memorias del VIII Encuentro Nacional de Experiencias en Enseñanza de la Biología y la Educación Ambiental. III Congreso Nacional de Investigación en Enseñanza de la Biología.

inventarse desde la singularidad de las prácticas de los maestros en donde se hace visible su ejercicio ético, político y estético en relación con la enseñanza.

En esa vía, a continuación se presentan una suerte de relaciones entre la enseñanza de lo vivo y la vida a propósito de la biología en tanto saber y las condiciones que se recrean a partir de ésta para pensar la constitución de los sujetos desde la estética; tomando elementos del diálogo que se establece entre dos trabajos de grado que presentan aspectos desde dos momentos históricos distintos. De acuerdo con ello en el primer apartado, se visibiliza un panorama general de la enseñanza de lo vivo y la vida a partir de elementos que se movilizaron en la escuela colombiana entre 1930 y 1940; en el segundo apartado, se establecen algunas relaciones entre la estética y la formación de maestros de biología, y se finaliza con algunas líneas de comprensión que apuntan a la emancipación de alternancias que permiten re-pensar la enseñanza de lo vivo y la vida en la escuela.

\section{METODOLOGÍA}

Este tejido escritural se constituye desde elementos de la caja de herramientas propuesta por Michel Foucault. Así se retoma la apuesta arqueológica - genealógica como una posibilidad para problematizar lo que se considera como verdad e interrogar las condiciones que han hecho posible que estas funcionen como moduladores de subjetividad. En esa medida, la mirada metodológica no pretende evidenciar las relaciones causa efecto, ni establecer interpretaciones de los discursos, por el contrario, interesa aquí mostrar el funcionamiento de los mismos.

Desde esta perspectiva, el situarse desde lo arqueológico-genealógico implica trabajar desde tres conceptos metodológicos: el saber, el poder y la subjetivación. Igualmente, se incita a la conformación del archivo donde los documentos son asumidos como prácticas, siendo éstos de cualquier índole pues más allá de la urgencia por el conocimiento y el lugar del experto, interesa visibilizar el saber que circula, es decir los sujetos que hablan, lo que dicen y qué instituciones lo respaldan. En ese sentido, la constitución del archivo implica el rastreo de documentos en diferentes periodos de acuerdo con las relaciones planteadas, para el caso se ha venido conformando por documentos desde el año 1930 a 1940 y del año 2000 a 2012, dichos documentos aluden a políticas, libros de texto, noticias, programas de formación, cuadernos de campo, escritos de maestros, entrevistas a maestros del DBI, entro otros, los cuales son tematizados, es decir, desarticulados en temas que se van organizando en una matriz y que al ser leída en distintos sentidos, posibilita identificar las regularidades y las discontinuidades a partir de las cuales se problematiza y se configura el objeto dejando entrever las relaciones que se tejen.

\section{LA FORMACIÓN ESTÉTICA EN BIOLOGÍA EN LA ESCUELA COLOMBIANA: UNA}


Bio -grafía. Escritos sobre la Biología y su Enseñanza. ISSN 2027-1034

\title{
Edición Extraordinaria. p.p.1057-1066
}

Memorias del VIII Encuentro Nacional de Experiencias en Enseñanza de la Biología y la Educación Ambiental. III Congreso Nacional de Investigación en Enseñanza de la Biología.

\section{MIRADA ENTRE 1930 Y 1940}

\begin{abstract}
"La naturaleza nos brinda en las plantas y en las flores sus más bellas y preciosas galas, como un gigantesco bouquet, cargado de perfumes y de vistosos colores. A esa gloria sin par, a ese imponderable presente de Dios, sepamos recibirlo como un marco de nuestro sentimiento de amantes de lo bello."
\end{abstract}

(Guzmán, 1938)

Los elementos que se presentan en este apartado se constituyen en una pregunta por las posibilidades que brinda la estética en la enseñanza de lo vivo y la vida desde una perspectiva histórica, entendiendo aquí la historia no como linealidad sino como rupturas que dan cuenta de discontinuidades, una historia que tiende más a una genealogía. Desde esa perspectiva, se visibilizan aspectos en relación con la formación estética en biología para los años en mención, encontrando en primera medida que la relación de los sujetos con la naturaleza se ve atravesada por elementos que pasan por el arte, la admiración y la sensibilidad. Otros matices, que impulsan la enseñanza de lo vivo y la vida no sólo desde el ideal de explotación de los recursos naturales, sino desde relaciones que incitan a ver en la naturaleza una fuente inagotable de bellezas dignas de ser cuidadas y valoradas, una naturaleza referida como la más bella creación de Dios.

Esta aproximación a la naturaleza se ve plasmada en libros de textos de la época en donde se ha dado paso a diversas composiciones de orden lírico donde priman algunas líneas inspiradas en la belleza de las plantas tal como se muestra: "La flor, esa mimada de los amantes, a la que enriquecieron el calor y la luz con especiales aromas, colores y belleza, no es, ni más ni menos, que un aéreo y efímero tálamo que naturaleza soñadora instaló en una gruta de hojas para los desposorios de dos seres que en el portentoso desarrollo de la vida nacieron para amarse y llevan en sus organismos el vigor de mil generaciones." (Uribe, 1930, p. 19)

Otro aspecto a destacar de los hallazgos es que el estudio y enseñanza de lo vivo y la vida desde lo estético está dirigida principalmente al niño pues "qué camino más seguro para infundir en los niños un profundo amor a la naturaleza que el llevarlos a su interpretación y hacer que ellos mismos indaguen y descubran sus fuerzas estéticas?" (Solano, 1934, p. 354). Un niño considerado como sujeto principal en los procesos educativos, por lo que es deber de la escuela y del maestro acercarlo al conocimiento de la naturaleza destacando la sensibilidad y el sentir de lo bello inspirado por ésta: "EI maestro que se ha observado transmitirá el don de la observación a sus alumnos, enseñándoles la belleza no solo con la palabra, sino con la representación misma de lo bello fuera de la escuela, la belleza natural que es la más genuina." (Froebel, 1925, p. 237).

Dirección bajo la cual se hacen presentes algunas consideraciones a propósito de la formación del niño campesino o de región rural, donde no solo encuentra la fuente de 


\section{Edición Extraordinaria. p.p.1057-1066}

Memorias del VIII Encuentro Nacional de Experiencias en Enseñanza de la Biología y la Educación Ambiental. III Congreso Nacional de Investigación en Enseñanza de la Biología.

ocupación laboral y riqueza económica, sino también la oportunidad de descubrir elementos que le proporcionen goces que alimenten su espíritu y que le permitan hacer más amena su vida: "Amor a la naturaleza y conocimiento de la flora y la fauna y sus relaciones con el hombre. Los niños de las regiones rurales deben tener conocimiento, en guarda de su formación estética, del goce que proporcionan los encantos de la naturaleza, de su armonía y su belleza, cuya comprensión emana en gran parte de los conocimientos íntimos de Natura, que dan a un caudal de virilidad, de energías y de satisfacción por los trabajos de la vida del campo." (Solano, 1934, p. 354).

Por esta vía conviene interrogar acerca de las prácticas en las que se materializa la formación estética en la escuela, ¿cuáles son éstas?. Desde lo que dejan entrever los documentos se posiciona el dibujo natural como un medio a través del cual es posible potenciar el acercamiento de los niños a su medio natural, máxime cuando se le ubica como una de las principales actividades en la enseñanza de las ciencias naturales en general "Es el dibujo una de las actividades más importantes en la enseñanza de las ciencias naturales. A medida que los niños observan es muy conveniente que se ejerciten en el dibujo del natural. No importa que los dibujos no sean tan perfectos como los quisiera el maestro, basta recordar que por más imperfectos que parezcan, para el niño representan la expresión de sus ideas." (Vargas, 1944, 159). Podría afirmarse que es por ello que la enseñanza del dibujo se encuentra instaurada en la escuela a tal punto que "en los grupos de tercero y cuarto dan buen resultados el que los alumnos lleven un cuaderno de dibujo natural destinado a las Ciencias Naturales." (Vargas, 1944, p. 160) La práctica de dibujar persigue diversos fines, por un lado capacitar al estudiante para servirse del dibujo como un medio de expresión, por el otro el constituirse como un fin educativo en el que el alumno desenvuelva sus actividades mentales, desarrolle sus sentidos y estimule el amor por lo bello.

En este sentido es viable pensar el dibujo como una actividad singular del sujeto en donde éste da paso a múltiples expresiones que nacen a partir de aquella experiencia suscitada por la observación, por lo que: “... el dibujo deberá ser también algo que se vive en el propio cuerpo y cada línea que se ha comprendido en un movimiento personal, cada recta cuya rigidez hemos establecido con una determinada posición nuestra y cada curva que nos ha llevado a una flexión, permanecerá indeleble en nuestro cerebro y en nuestro sentimiento con toda la belleza de su expresión honda." (Cock, 1933, p. 151) . Así en relación con la educación estética a propósito de lo natural, el objetivo de la escuela no es formar eruditos en dicho campo, antes bien se propone crear las condiciones necesarias para que el sujeto sea capaz de sentir verdaderamente la belleza de su medio. De esta manera se considera importante dirigir la mirada a la formación de maestros, en la que se incorporan elementos en relación a la preparación estética: "Aspiremos a que nuestra Facultad de Educación pueda dar con el tiempo a sus alumnos esa preparación estética tan indispensable en los verdaderos educadores." (Cock, 1933, 


\section{Edición Extraordinaria. p.p.1057-1066}

Memorias del VIII Encuentro Nacional de Experiencias en Enseñanza de la Biología y la Educación Ambiental. III Congreso Nacional de Investigación en Enseñanza de la Biología.

p. 154)

\section{HILOS DE RELACIÓN ENTRE LA ESTÉTICA Y LA FORMACIÓN DE MAESTROS DEL DEPARTAMENTO DE BIOLOGÍA DE LA UPN}

A continuación se pretende visibilizar un haz de relaciones que permite dar despliegue tanto a la estética como a la formación de maestros de biología, no sin antes desconocer que en el panorama general se sitúa la estética de lado del aprendizaje a propósito de la formación de sujetos productivos y competentes en términos de las demandas del mercado dentro del mundo contemporáneo. En esa vía, el maestro debe orientar los procesos de enseñanza-aprendizaje de sus estudiantes hacia el aprovechamiento de las actividades que realice en pro de una formación estética, entendida como parte del desarrollo subjetivo del estudiante, de su placer estético, de la capacidad para apreciar y comprender la belleza dentro de la realidad; así por ejemplo: "el profesor de biología, cuya función es siempre el cuidado de las sensaciones humanas para la vida y por lo vivo, endilgará a sus alumnos hacia la utilidad de lo bello que existe en la naturaleza y su creación [...]" (Bandera y Espinoza, 2011, p.5)

En relación con la anterior cita, surge el interrogante: ¿es posible que los maestros de biología cuenten con una formación estética?, al respecto, Hernández (1999) asegura que puede haber cierta claridad en cómo formar al maestro en el campo del saber y el saber-hacer, pero en cuanto al terreno de la estética, resulta confuso y prácticamente inaccesible; por tanto, pensar en una formación estética desde las dinámicas del mundo actual, reafirma el lugar de la belleza y la sensibilidad como materias constructoras de nuevas formas de entender el mundo desde la heterogeneidad en que se nos presenta, para ello, formar a los maestros desde una perspectiva estética implica el desarrollo de competencias y de flexibilidad ante ambientes de aprendizaje polisémicos. Es así que la estética, ha incursionado en el discurso de las competencias como objeto de intereses y propósitos determinados, como herramienta útil y eficaz en ciertos momentos de la práctica del maestro, según Jiménez y Calderón (2005), la competencia estética alude al deseo y la voluntad del saber del maestro, al placer y la capacidad de conocer comunicar y estimular el trabajo de los estudiantes, pero también, de crear alternativas frente a las dificultades que se ciernen dentro y fuera del aula.

Así mismo, las relaciones con la estética parten de discursos laudatorios que hacen mención al maestro como un sujeto "estético", los cuales, pareciera que se han convertido en un fetiche en tanto se deben buscar, reconocer y elaborar. Pero ¿Por qué pensar en el maestro como sujeto estético?, más allá de una afirmación, esta posibilidad emerge desde los modos de ser y estar del maestro en el mundo, de habitar la formasujeto con miras a su configuración. $Y$ es que precisamente, estos nuevos modos no refieren ni a las demandas ni a las exigencias que se le atribuyen, sino más bien, a la 


\section{Edición Extraordinaria. p.p.1057-1066}

Memorias del VIII Encuentro Nacional de Experiencias en Enseñanza de la Biología y la Educación Ambiental. III Congreso Nacional de Investigación en Enseñanza de la Biología.

potencia de su voz, la plasticidad de su cuerpo, la teatralidad de su oralidad, y su gesto pedagógico, es así que la estética del maestro no se compone de la belleza de sus actos, pero sí, de la fuerza de novedad de su pensamiento creativo.

Ahora bien, al situarnos en la formación de maestros de biología, concretamente, en las experiencias de los maestros, se encuentra que la relación biología-estética, permite un deslocalizamiento en doble vía: la Biología, del objeto disciplinar demarcado por prácticas científicas y utilitaristas que propugnan la solución de problemáticas relevantes en relación con el cuidado y protección tanto del medio ambiente como del cuerpo; y la estética, como una disciplina enfatizada en dinámicas de perfección y belleza, o en el caso de la escuela, como una eficaz herramienta para aproximar a los estudiantes hacia el conocimiento de los contornos biológicos. Así, dicha deslocalización nos permite aproximarnos a la configuración de las relaciones que establecemos con el mundo: consigo mismo, con los demás, con lo vivo y la vida, desde prácticas sensibles matizadas de afección ${ }^{4}$ como vía hacia el pensamiento.

Pero entonces, ¿Cuáles son aquellas condiciones que hacen posible esta deslocalización? en primera instancia, el archivo deja ver la práctica pedagógica y la investigación como una experiencia de formación ampliamente nutrida por prácticas escriturales desde el cuaderno de campo donde se conjugan trazos que hacen alusión al dibujo científico y a una escritura que si bien tiene que ver con datos biológicos, éstos se hallan interrelacionados con el sentir del maestro frente a lo que acontece en el ámbito socio-cultural a propósito de prácticas que se movilizan en distintos escenarios como las salidas de campo; y prácticas singulares que tienen lugar desde el devenir del maestro de Biología, es decir, aquello que ha dado emergencia a un modo de vida singular, distante de las funciones y deberes delegadas, más próximo a pensar en la configuración del maestro y de la Biología, no como relación de causa-efecto que trae consigo la imprescindible utilidad de la relación vivo-vida, sino más bien, como relación que da apertura a una fuerza de novedad cuyos tintes apuntan a dilucidar otras ópticas que dialogan con lo vivo y la vida, tendientes incluso, a recomponer y modificar las relaciones del maestro con su saber, al reconocerse más que como funcionario de los compromisos contractuales, como maestro que denota preocupación por el sujeto.

Estas condiciones han posibilitado la emergencia de unas prácticas de sí que sitúan al maestro de biología como un creador de posibilidades vitales de existencia en su práctica pedagógica, allí, la investigación no se agota en la obligación pues pasa por el gusto y la inquietud propia, pasa por la escritura, la biología, el arte, la pedagogía y los escenarios, en definitiva, este conglomerado de relaciones toca la vida de los maestros,

\footnotetext{
${ }^{4}$ Desde los tránsitos investigativos se asume la afección como la problematización constante de aquello que el sujeto confronta en su cotidianidad.
} 


\section{Edición Extraordinaria. p.p.1057-1066}
Memorias del VIII Encuentro Nacional de Experiencias en Enseñanza de la Biología y la Educación Ambiental. III Congreso Nacional de Investigación en Enseñanza de la Biología.

desde ese lugar de pasaje y conmoción las relaciones se recomponen, los pensamientos y sentimientos se movilizan, lo estable se hace inestable y el otro, se convierte en posibilidad de construir, problematizar, compartir y vivir en conjunto. Habría que decir también, que los maestros de biología no se sienten ajenos a la estética, pues el trasegar de su experiencia les ha permitido deslocalizar ese estatus legítimo de perfección y belleza o la relevancia en tanto estrategia para el aprendizaje de la biología, de esta manera, la enseñanza de lo vivo y la vida es quizá una práctica estética que desborda los contenidos que son objeto de aprendizaje puesto que tendría que ver con los posicionamientos que configuramos frente a lo vivo y la vida, con una estilística del vivir que al no estar predeterminada es susceptible de re-inventarse.

\section{ALGUNAS CONSIDERACIONES...}

Las líneas aquí expuestas pretenden hacer una invitación a re-pensar las prácticas relacionadas con la enseñanza de lo vivo y la vida en tanto potencia del maestro, precisamente, su experiencia posibilita configurar prácticas singulares que si bien emergen de las relaciones que se establecen frente al mundo, no se hallan supeditadas a la funcionalidad de su quehacer. En este sentido, la estética es una de las condiciones que hace posible la emancipación de prácticas de sí que ponen el acento en la formación, distando de las lógicas del saber responder contractual y aproximada más bien, hacia un pasaje de afecto y conmoción por configurar desde un trasegar propio.

\section{BIBLIOGRAFÍA}

- Bandera, Anselmo y Espinoza, Enrique. Lo estético en el proceso docente en la formación integral del profesional universitario. Cuba. En: http://www.monografias.com/trabajos75/estetica-proceso-docenteprofesional-universitario/estetica-proceso-docente-profesional-universitario2.shtml

- Crock, L. (1933). La educación estética en la escuela. En: Revista Educación. Año 1. Número 3. Facultad de Educación de la Universidad Nacional de Colombia. Bogotá

- Froebel, F. (1925). El huerto-jardín escolar: su valor social y económico. En Revista Nacional de Agricultura vol. 19 no. 249 - 250. Bogotá

- Guzmán, A. (1938). Amor a las plantas. En Revista Nacional de Agricultura. No. 413. 1938. Editorial Centro S.A. Bogotá.

- Hernández, Carlos (1999). Aproximaciones a la discusión sobre el perfil docente. Disponible en: http://www.oei.org.co/de/cah.htm. Bogotá.

- Jiménez, Ana; Ariza, Carmenza; Calderón, Stella (2005). La visión humanista del maestro un desafío frente a su formación. En: Formación de maestros, profesión y trabajo docente. 2005, Bogotá: Universidad Pedagógica Nacional. p.76-81.

- Martínez, Alberto (2003). La enseñanza como posibilidad de pensamiento. En: Pedagogía y Epistemología. Bogotá: Cooperativa Editorial Magisterio.

- Peñuela, Diana (2010). El Arte de resistir desde la pedagogía y la condición humana del maestro. Revista Cuestiones de Filosofía, No.12. Bogotá: Universidad Pedagógica y Tecnológica de Colombia. p. 1-14

- Solano, N. (1934). La escuela rural en Colombia. En: Revista Educación. Año 1. Número 11. Facultad de Educación de la Universidad Nacional de Colombia. Bogotá.

- Uribe, J. (1930). Curso compendiado de historia natural. Tipografía Bedout. Medellín

- Vargas, C. (1944). Botánica elemental para escuela primaria. Editorial Minerva. Bogotá 